\title{
Research on a Class of Fourth-order Rouge-Kutta Algorithm for Real-Time Simulation
}

\author{
Zhen-Quan Huang ${ }^{*}$, Su-Ying Liao, Yun He and Ai-Jun Tang \\ Air Force Airborne Academy, Guilin, China \\ hzq922@163.com
}

\begin{abstract}
Keywords: Real-time simulation, Runge-Kutta (RK) algorithm, Parametric equations, The coefficients of truncation error.

Abstract. From the basic idea of the real-time Runge-Kutta (RK) algorithm, some parametric equations satisfied the general form of the real-time fourth-order RK algorithm are derived. Then the coefficients of truncation error are deduced based on the analysis for local truncation errors of real-time RK algorithm. Finally a new formula of the real-time RK algorithm is proposed according to the related equations of the stability region and truncation errors. The new algorithm formula would distinctly reduce the truncation errors for some real-time simulation questions, thus it can be applied in a extensive area.
\end{abstract}

\section{Introduction}

Initial value problem of ordinary differential equation often occurs in real-time simulation, where related real-time algorithm must be taken. The RK4 algorithm, with the advantage of self-starting and easy access to error estimation, along with the fact that fourth-order accuracy can be accept in most engineering problems, has become a typical numerical calculation method in real-time simulation.

$$
\dot{y}(x)=f[x, y(x), u(x)] \quad y\left(x_{0}\right)=y_{0}
$$

Since the RK algorithm is an approximate algorithm based on Taylor Series, it's necessary to select one real-time RK algorithm with small truncation error to meet the demand of real-time performance. The common RK algorithm are described in detail in [1-3], while in this paper the real-time RK algorithm is discussed, the parametric equations are determined, and a new real-time RK4 algorithm is derived. The results might have important theoretical significance and can be applied in an extensive way.

\section{Parametric equations of the real-time RK4 algorithm}

The single-step explicit formula of RK algorithm is extensively adopted in real-time simulation. As the order and the coefficients differ, it has different forms.

To meet the demand of real-time performance, for s level low order RK algorithm, the input sampling interval is set to $\Delta t=h / \mathrm{s}$, the output interval is set to $\Delta t=h$, including RTRK1 algorithm, RTRK2 algorithm and RTRK3 algorithm.

As to non real-time fourth-order RK algorithm, for example the classical four level fourth-order RK formula, there exists no one satisfying real-time performance from[4], so here we look for a five level fourth-order real-time RK formula, of which the general form is

$$
\left\{\begin{aligned}
y_{n+1} & =y_{n}+h \sum_{i=1}^{5} c_{i} k_{i} \\
k_{i} & =f\left[x_{n}+a_{i} h, y_{n}+h \sum_{j=1}^{i-1} b_{i j} k_{j}, u\left(x_{n}+a_{i} h\right)\right] \\
a_{i} & =\frac{i-1}{5} \quad i=1,2,3,4,5
\end{aligned}\right.
$$

For the convenience of sampling, the input interval is $\Delta t=h / 5$, thus $a_{i}=0,1 / 5,2 / 5,3 / 5,4 / 5$.

The function $f(x, y)$ in ODE (1) can be expanded by Taylor Series of binary functions as follows ${ }^{[5]}$ 


$$
\begin{aligned}
& f\left(x_{0}+h, y_{0}+k\right)=f\left(x_{0}, y_{0}\right)+\left(h \frac{\partial}{\partial x}+k \frac{\partial}{\partial y}\right) f\left(x_{0}, y_{0}\right)+\frac{1}{2 !}\left(h \frac{\partial}{\partial x}+k \frac{\partial}{\partial y}\right)^{2} f\left(x_{0}, y_{0}\right)+\cdots+\frac{1}{n !}\left(h \frac{\partial}{\partial x}+k \frac{\partial}{\partial y}\right)^{n} f\left(x_{0}, y_{0}\right) \\
& +\frac{1}{(n+1) !}\left(h \frac{\partial}{\partial x}+k \frac{\partial}{\partial y}\right)^{n+1} f\left(x_{0}+\theta h, y_{0}+\theta k\right) \quad(0<\theta<1)
\end{aligned}
$$

To make our derivations easier, let $D=\frac{\partial}{\partial x}+f \frac{\partial}{\partial y}$.

The basic idea of RK4 algorithm is trying to forecast the slopes $k_{i}$ of five points in $\left[x_{n}, x_{n+1}\right]$ and taking the weighted average as the average slope $k^{*}$ to obtain a formula with higher degrees of accuracy. According to Taylor's expansion

$$
\begin{aligned}
& y\left(x_{0}+h\right)=y\left(x_{0}\right)+h y^{\prime}+\frac{1}{2 !} h^{2} y^{\prime \prime}+\frac{1}{3 !} h^{3} y^{\prime \prime \prime}+\cdots+\frac{1}{n !} h^{n} y^{n}+\cdots=y\left(x_{0}\right)+\left[h f+\frac{h^{2}}{2 !} D f+\frac{h^{3}}{3 !}\left(D^{2} f+f_{y} D f\right)+\frac{h^{4}}{4 !}\left(D^{3} f+f_{y} D^{2} f\right.\right. \\
& \left.\left.+f_{y}^{2} D f+3 D f D f_{y}\right)+\frac{h^{5}}{5 !}\left(D^{4} f+6 D f D^{2} f_{y}+4 D^{2} f D+D^{2} f f_{y}^{2}+D f f_{y}^{3}+3(D f)^{2} f_{y y}+D^{3} f f_{y}+7 f_{y} D f D f_{y}\right)+\cdots\right]
\end{aligned}
$$

On the basis of the idea of real-time RK4 algorithm we have

$$
\begin{aligned}
& a_{2}=b_{21}=1 / 5 \\
& a_{3}=b_{31}+b_{32}=2 / 5 \\
& a_{4}=b_{41}+b_{42}+b_{43}=3 / 5 \\
& a_{5}=b_{51}+b_{52}+b_{53}+b_{54}=4 / 5
\end{aligned}
$$

To make derivations easier, let

$$
\begin{aligned}
& D_{1}=a_{2} \frac{\partial}{\partial x}+b_{21} f \frac{\partial}{\partial y}=a_{2} D \\
& D_{2}=a_{3} \frac{\partial}{\partial x}+\left(b_{31}+b_{32}\right) f \frac{\partial}{\partial y}=a_{3} D \\
& D_{3}=a_{4} \frac{\partial}{\partial x}+\left(b_{41}+b_{42}+b_{43}\right) f \frac{\partial}{\partial y}=a_{4} D \\
& D_{4}=a_{5} \frac{\partial}{\partial x}+\left(b_{51}+b_{52}+b_{53}+b_{54}\right) f \frac{\partial}{\partial y}=a_{5} D \\
& D_{11}=a_{2} h \frac{\partial}{\partial x}+b_{21} k_{1} \frac{\partial}{\partial y}=a_{2} h \frac{\partial}{\partial x}+b_{21} h f \frac{\partial}{\partial y}=h D_{1}=h a_{2} D \\
& D_{21}=a_{3} h \frac{\partial}{\partial x}+\left(b_{31} k_{1}+b_{32} k_{2}\right) \frac{\partial}{\partial y}=h\left(a_{3} \frac{\partial}{\partial x}+b_{31} f \frac{\partial}{\partial y}\right)+b_{32} k_{2} \frac{\partial}{\partial y}=h D_{2}+b_{32}\left(k_{2}-h f\right) \frac{\partial}{\partial y} \\
& =h D_{2}+h^{2} b_{32}\left(D_{1} f+\frac{h}{2 !} D_{1}^{2} f+\frac{h^{2}}{3 !} D_{1}^{3} f+\cdots\right) \frac{\partial}{\partial y}=h a_{3} D+h^{2} b_{32}\left(a_{2} D f+\frac{h}{2 !} a_{2}^{2} D^{2} f+\frac{h^{2}}{3 !} a_{2}^{3} D^{3} f+\cdots\right) \frac{\partial}{\partial y} \\
& D_{31}=a_{4} h \frac{\partial}{\partial x}+\left(b_{41} k_{1}+b_{42} k_{2}+b_{43} k_{3}\right) \frac{\partial}{\partial y}=h D_{3}+\left[b_{42}\left(k_{2}-h f\right)+b_{43}\left(k_{3}-h f\right)\right] \frac{\partial}{\partial y} \\
& =h D_{3}+h^{2}\left[b_{42}\left(D_{1} f+\frac{h}{2 !} D_{1}^{2} f+\frac{h^{2}}{3 !} D_{1}^{3} f+\cdots\right)+b_{43}\left(D_{2} f+\frac{h}{2 !} D_{2}^{2} f+2 b_{32} f_{y} D_{1} f\right)+\frac{h^{2}}{3 !} D_{2}^{3} f+\frac{h^{2}}{2 !} b_{32} f_{y} D_{1}^{2} f+h^{2} b_{32} D_{1} f D_{2} f_{y}+\cdots\right] \frac{\partial}{\partial y} \\
& D_{41}=a_{5} h \frac{\partial}{\partial x}+\left(b_{51} k_{1}+b_{52} k_{2}+b_{53} k_{3}+b_{54} k_{4}\right) \frac{\partial}{\partial y}=h D_{4}+\left[b_{52}\left(k_{2}-h f\right)+b_{53}\left(k_{3}-h f\right)+b_{54}\left(k_{4}-h f\right)\right] \frac{\partial}{\partial y}=h D_{4}+h^{2}\left\{b _ { 5 2 } \left(D_{1} f+\frac{h}{2 !} D_{1}^{2} f\right.\right. \\
& \left.+\frac{h^{2}}{3 !} D_{1}^{3} f+\cdots\right)+b_{53}\left[D_{2} f+\frac{h}{2 !}\left(D_{2}^{2} f+2 b_{32} f_{y} D_{1} f\right)+\frac{h^{2}}{3 !} D_{2}^{3} f+\frac{h^{2}}{2 !} b_{32} f_{y} D_{1}^{2} f+h^{2} b_{32} D_{1} f D_{2} f_{y}+\cdots\right]+b_{54}\left\{D_{3} f+h f_{y}\left[b _ { 4 2 } \left(D_{1} f\right.\right.\right. \\
& \left.\left.+\frac{h}{2} D_{1}^{2} f+\cdots\right)+b_{43}\left(D_{2} f+h b_{32} f_{y} D_{1} f+\frac{h}{2} D_{2}^{2} f+\cdots\right)\right]+\frac{1}{2 !}\left\{h D_{3}^{2} f+2 h^{2} D_{3} f_{y}\left[b_{42}\left(D_{1} f+\cdots\right)+b_{43}\left(D_{2} f+\cdots\right)\right]+\cdots\right\}+\frac{h^{2}}{3 !} D_{3}^{3} f \\
& +\cdots\}\} \frac{\partial}{\partial y} \\
& =h a_{5} D+h^{2}\left\{b_{52}\left(a_{2} D f+\frac{h}{2 !} a_{2}^{2} D^{2} 2 f+\frac{h^{2}}{3 !} a_{2}^{3} D^{3} f+\cdots\right)+b_{53}\left[a_{3} D f+\frac{h}{2 !}\left(a_{3}^{2} D^{2} f+2 b_{32} a_{2} f_{y} D f\right)+\frac{h^{2}}{3 !} a_{3}^{3} D^{3} f+\frac{h^{2}}{2 !} b_{32} a_{2}^{2} f_{y} D^{2} f\right.\right. \\
& \left.+h^{2} b_{32} a_{2} a_{3} D f D f_{y}+\cdots\right]+b_{54}\left\{a_{4} D f+h f_{y}\left[b_{42}\left(a_{2} D f+\frac{h}{2} a_{2}^{2} D^{2} f+\cdots\right)+b_{43}\left(a_{3} D f+h b_{32} a_{2} f_{y} D f+\frac{h}{2} a_{3}^{2} D^{2} f+\cdots\right)\right]+\frac{1}{2 !}\left\{h a_{4}^{2} D^{2} f\right.\right. \\
& \left.\left.\left.+2 h^{2} a_{4} D f_{y}\left[b_{42}\left(a_{2} D f+\cdots\right)+b_{43}\left(a_{3} D f+\cdots\right)\right]+\cdots\right\}+\frac{h^{2}}{3 !} a_{4}^{3} D^{3} f+\cdots\right\}\right\} \frac{\partial}{\partial y}
\end{aligned}
$$

Expand $k_{2}, k_{3}, k_{4}, k_{5}$ at $\left(x_{n}, y_{n}\right)$ by using Taylor's expansion of binary functions; we can obtain the following results after some time-consuming calculations

$k_{1}=h f$

$k_{2}=h\left(f+D_{11} f+\frac{D_{11}^{2}}{2 !} f+\frac{D_{11}^{3}}{3 !} f+\frac{D_{11}^{4}}{4 !} f+\cdots\right)=h\left(f+h a_{2} D f+\frac{h^{2}}{2 !} a_{2}^{2} D^{2} f+\frac{h^{3}}{3 !} a_{2}^{3} D^{3} f+\frac{h^{4}}{4 !} a_{2}^{4} D^{4} f+\cdots\right)$ 
$k_{3}=h\left(f+D_{21} f+\frac{D_{21}^{2}}{2 !} f+\frac{D_{21}^{3}}{3 !} f+\frac{D_{21}^{4}}{4 !} f+\cdots\right)$

$=h\left\{f+h a_{3} D f+\frac{h^{2}}{2 !} a_{3}^{2} D^{2} f+\frac{h^{3}}{3 !} a_{3}^{3} D^{3} f+\frac{h^{4}}{4 !} a_{3}^{4} D^{4} f+\cdots+h^{2} b_{32}\left[f_{y} a_{2} D f+h a_{2} a_{3} D f D f_{y}+\frac{h}{2 !} a_{2}^{2} f_{y} D^{2} f+\frac{h^{2}}{3 !} a_{2}^{3} f_{y} D^{3} f\right.\right.$

$\left.\left.+\frac{h^{2}}{2 !} a_{2}^{2} a_{3} D^{2} f D f_{y}+\frac{h^{2}}{2 !} b_{32} a_{2}^{2} f_{y y}(D f)^{2}+\frac{h^{2}}{2 !} a_{2} a_{3}^{2} D f D^{2} f_{y}+\cdots\right]\right\}$

$k_{4}=h\left(f+D_{31} f+\frac{D_{31}^{2}}{2 !} f+\frac{D_{31}^{3}}{3 !} f+\frac{D_{31}^{4}}{4 !} f+\cdots\right)$

$=h\left\{f+h a_{4} D f+h^{2} f_{y}\left[b_{42}\left(a_{2} D f+\frac{h}{2} a_{2}^{2} D^{2} f+\frac{h^{2}}{3 !} a_{2}^{3} D^{3} f+\cdots\right)+b_{43}\left(a_{3} D_{2} f+h b_{32} a_{2} f_{y} D f+\frac{h}{2} a_{3}^{2} D^{2} f+\frac{h^{2}}{2} b_{32} a_{2} f_{y} D^{2} f+h^{2} b_{32} a_{2} a_{3} D f D f_{y}\right.\right.\right.$

$\left.\left.+\frac{h^{3}}{3 !} a_{3}^{3} D^{3} f+\cdots\right)\right]+\frac{1}{2 !}\left\{h^{2} a_{4}^{2} D^{2} f+2 h^{3} a_{4} D f_{y}\left[b_{42}\left(a_{2} D f+\frac{h}{2} a_{2}^{2} D^{2} f+\cdots\right)+b_{43}\left(a_{3} D f+h b_{32} a_{2} f_{y} D f+\frac{h}{2} a_{3}^{2} D^{2} f+\cdots\right)\right]+h^{4} f_{y y}\left[b_{42}^{2}\left(a_{2} D f\right)^{2}\right.\right.$

$\left.\left.\left.+2 b_{42} b_{43} a_{2} a_{3}(D f)^{2}+\left(b_{43} a_{3}\right)^{2}(D f)^{2}+\cdots\right]\right\}+\frac{1}{3 !}\left[h^{3} a_{4}^{3} D^{3} f+3 h^{4} a_{4}^{2} D^{2} f_{y}\left(b_{42} a_{2} D f+b_{43} a_{3} D f+\cdots\right)\right]+\frac{1}{4 !}\left(h^{4} a_{4}^{4} D^{4} f+\cdots\right)+\cdots\right\}$

$k_{5}=h\left(f+D_{41} f+\frac{D_{41}^{2}}{2 !} f+\frac{D_{41}^{3}}{3 !} f+\frac{D_{41}^{4}}{4 !} f+\cdots\right)$

$=h\left\{f+h a_{5} D f+h^{2} f_{y}\left\{b_{52}\left(a_{2} D f+\frac{h}{2 !} a_{2}^{2} D^{2} f+\frac{h^{2}}{3 !} a_{2}^{3} D^{3} f+\cdots\right)+b_{53}\left[a_{3} D f+\frac{h}{2 !}\left(a_{3}^{2} D^{2} f+2 b_{32} a_{2} f_{y} D f\right)+\frac{h^{2}}{3 !} a_{3}^{3} D^{3} f+\frac{h^{2}}{2 !} b_{32} a_{2}^{2} f_{y} D^{2} f\right.\right.\right.$

$\left.+h^{2} b_{32} a_{2} a_{3} D f D f_{y}+\cdots\right]+b_{54}\left\{a_{4} D f+h f_{y}\left[b_{42}\left(a_{2} D f+\frac{h}{2 !} a_{2}^{2} D^{2} f+\cdots\right)+b_{43}\left(a_{3} D f+h b_{32} a_{2} f_{y} D f+\frac{h}{2 !} a_{3}^{2} D^{2} f+\cdots\right)\right]+\frac{1}{2 !}\left[h a_{4}^{2} D^{2} f\right.\right.$

$\left.\left.\left.+2 h^{2} a_{4} D f_{y}\left(b_{42} a_{2} D f+b_{43} a_{3} D f+\cdots\right)+\cdots\right]+\frac{1}{3 !}\left(h^{2} a_{4}^{3} D^{3} f+\cdots\right)+\cdots\right\}\right\}+\frac{1}{2 !}\left\{h a_{5}^{2} D^{2} f+2 h^{3} a_{5} D f_{y}\left\{b_{52}\left(a_{2} D f+\frac{h}{2 !} a_{2}^{2} D^{2} f+\cdots\right)\right.\right.$

$\left.+b_{53}\left[a_{3} D f+\frac{h}{2}\left(a_{3}^{2} D^{2} f+2 b_{32} a_{2} f_{y} D f\right)+\cdots\right]+b_{54}\left[a_{4} D f+h f_{y}\left(b_{42} a_{2} D f+b_{43} a_{3} D f+\cdots\right)+\frac{h}{2 !} a_{4}^{2} D^{2} f+\cdots\right]\right\}+h^{4} f_{y y}\left[b_{52}^{2}\left(a_{2} D f\right)^{2}\right.$

$\left.\left.+2 b_{52} b_{53} a_{2} a_{3}(D f)^{2}+\left(b_{53} a_{3}\right)^{2}(D f)^{2}+b_{54}^{2}\left(a_{4} D f\right)^{2}+2 b_{53} b_{54} a_{3} a_{4}(D f)^{2}+2 b_{52} b_{54} a_{2} a_{4}(D f)^{2}+\cdots\right]+\cdots\right\}$

$\left.+\frac{1}{3 !} h^{3} a_{5}^{3} D^{3} f+\frac{1}{2 !} h^{4} a_{5}^{2} D^{2} f_{y}\left(b_{52} a_{2} D f+b_{53} a_{3} D f+b_{54} a_{4} D f+\cdots\right)+\cdots+\frac{1}{4 !} h^{4} a_{5}^{4} D^{4} f+\cdots\right\}$

By relations of $k_{1}, k_{2}, k_{3}, k_{4}, k_{5}$ we have

$y_{n+1}=y_{n}+c_{1} k_{1}+c_{2} k_{2}+c_{3} k_{3}+c_{4} k_{4}+c_{5} k_{5}$

$=y_{n}+\left(c_{1}+c_{2}+c_{3}+c_{4}+c_{5}\right) f h+\left(a_{2} c_{2}+a_{3} c_{3}+a_{4} c_{4}+a_{5} c_{5}\right) D f h^{2}+\left\{\left(a_{2}^{2} c_{2}+a_{3}^{2} c_{3}+a_{4}^{2} c_{4}+a_{5}^{2} c_{5}\right) \frac{D^{2} f}{2 !}\left[c_{3} b_{32} a_{2}+c_{4}\left(b_{42} a_{2}+b_{43} a_{3}\right)\right.\right.$

$\left.\left.+c_{5}\left(b_{52} a_{2}+b_{53} a_{3}+b_{54} a_{4}\right)\right] f_{y} D f\right\} h^{3}+\left\{\left(a_{2}^{3} c_{2}+a_{3}^{3} c_{3}+a_{4}^{3} c_{4}+a_{5}^{3} c_{5}\right) \frac{D^{3} f}{3 !}+\left[c_{3} b_{32} a_{2}^{2}+c_{4}\left(b_{42} a_{2}^{2}+b_{43} a_{2}^{3}\right)+c_{5}\left(b_{52} a_{2}^{2}+b_{53} a_{3}^{2}+b_{54} a_{4}^{2}\right)\right] \frac{f_{y} D^{2} f}{2 !}\right.$

$\left.+\left[c_{4} b_{32} b_{43} a_{2}+c_{5}\left(b_{32} b_{53} a_{2}+b_{42} b_{54} a_{2}+b_{43} b_{54} a_{3}\right)\right] f_{y}^{2} D f+\left[c_{3} b_{32} a_{2} a_{3}+a_{4} c_{4}\left(b_{42} a_{2}+b_{43} a_{3}\right)+a_{5} c_{5}\left(b_{52} a_{2}+b_{53} a_{3}+b_{54} a_{4}\right)\right] D f D f_{y}\right\} h^{4}$

$+\left\{\left(c_{2} a_{2}^{4}+c_{3} a_{3}^{4}+c_{4} a_{4}^{4}+c_{5} a_{5}^{4}\right) \frac{D^{4} f}{4 !}+\left[c_{3} b_{32} a_{2} a_{3}^{2}+c_{4}\left(b_{42} a_{2}+b_{43} a_{3}\right) a_{4}^{2}+c_{5}\left(b_{52} a_{2}+b_{53} a_{3}+b_{54} a_{4}\right) a_{5}^{2}\right] \frac{D f D^{2} f_{y}}{2}+\left[c_{4} a_{2}^{2} b_{32} b_{43}+c_{5}\left(a_{2}^{2} b_{32} b_{53}\right.\right.\right.$

$\left.\left.+a_{2}^{2} b_{42} b_{54}+a_{3}^{2} b_{43} b_{54}\right)\right] \frac{D^{2} f f_{y}^{2}}{2}+\left[c_{3} b_{32} a_{2}^{2} a_{3}+c_{4}\left(b_{42} a_{2}^{2}+b_{43} a_{3}^{2}\right) a_{4}+c_{5}\left(b_{52} a_{2}^{2}+b_{53} a_{3}^{2}+b_{54} a_{4}^{2}\right) a_{5}\right] \frac{D^{2} f D f_{y}}{2}+c_{5} a_{2} b_{32} b_{43} b_{54} D f f_{y}^{3}+\left[c_{3} a_{2}^{2} b_{32}^{2}\right.$

$\left.+c_{4}\left(a_{2} b_{42}+a_{3} b_{43}\right)^{2}+c_{5}\left(a_{2} b_{52}+a_{3} b_{53}+a_{4} b_{54}\right)^{2}\right] \frac{1}{2}(D f)^{2} f_{y y}+\left[c_{3} a_{2}^{3} b_{32}+c_{4}\left(a_{2}^{3} b_{42}+a_{3}^{3} b_{43}\right)+c_{5}\left(a_{2}^{3} b_{52}+a_{3}^{3} b_{53}+a_{4}^{3} b_{54}\right)\right] \frac{1}{3 !} D^{3} f f_{y} \frac{D f D^{2} f_{y}}{2}$

$+\left[c_{4} a_{2}^{2} b_{32} b_{43}+c_{5}\left(a_{2}^{2} b_{32} b_{53}+a_{2}^{2} b_{42} b_{54}+a_{3}^{2} b_{43} b_{54}\right)\right] \frac{D^{2} f f_{y}^{2}}{2}+\left[c_{3} b_{32} a_{2}^{2} a_{3}+c_{4}\left(b_{42} a_{2}^{2}+b_{43} a_{3}^{2}\right) a_{4}+c_{5}\left(b_{52} a_{2}^{2}+b_{53} a_{3}^{2}+b_{54} a_{4}^{2}\right) a_{5}\right] \frac{D^{2} f D f_{y}}{2}$

$+c_{5} a_{2} b_{32} b_{43} b_{54} D f f_{y}^{3}+\left[c_{3} a_{2}^{2} b_{32}^{2}+c_{4}\left(a_{2} b_{42}+a_{3} b_{43}\right)^{2}+c_{5}\left(a_{2} b_{52}+a_{3} b_{53}+a_{4} b_{54}\right)^{2}\right] \frac{1}{2}(D f)^{2} f_{y y}+\left[c_{3} a_{2}^{3} b_{32}+c_{4}\left(a_{2}^{3} b_{42}+a_{3}^{3} b_{43}\right)\right.$

$\left.\left.+c_{5}\left(a_{2}^{3} b_{52}+a_{3}^{3} b_{53}+a_{4}^{3} b_{54}\right)\right] \frac{1}{3 !} D^{3} f f_{y}+\left\{c_{4} a_{2}\left(a_{3}+a_{4}\right) b_{32} b_{43}+c_{5}\left[a_{2}\left(a_{3}+a_{5}\right) b_{32} b_{53}+\left(a_{4}+a_{5}\right)\left(a_{2} b_{42}+a_{3} b_{43}\right) b_{54}\right]\right\} f_{y} D f D f_{y}\right\} h^{5}+\cdots$

Because the local truncation error of real-time RK4 algorithm is $O\left(h^{5}\right)$ and the accuracy is fourth-order, the equations satisfied by the parameters in the algorithm can be obtained by comparing the coefficients of $h 、 h^{2} 、 h^{3} 、 h^{4}$ in (4) and (14):

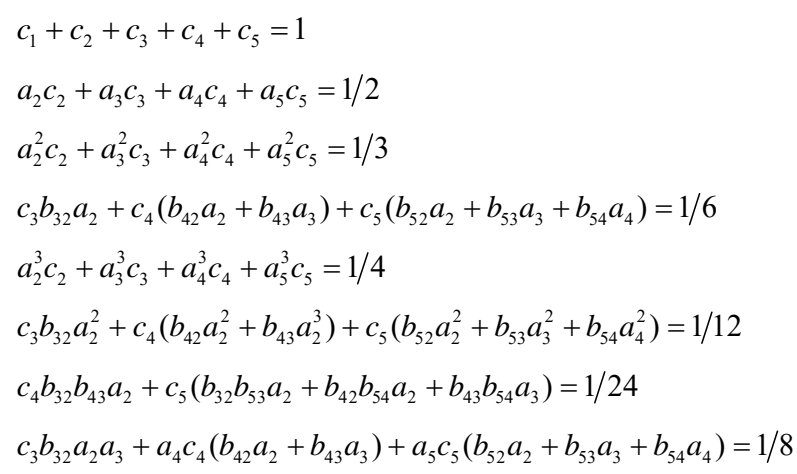

By the above analysis, (5)-(8) and (15)-(22) are the 12 parametric equations satisfied by the parameters in the general form of real-time RK4 algorithm. 


\section{A class of new real-time RK4 algorithm}

By analyzing the definite stability region of real-time RK4 algorithm we have ${ }^{[6]}$

$\alpha^{*}=b_{21} b_{32} b_{43} b_{54} c_{5}=0.00449$

The maximum area of definite stability region of real-time five level fourth-order RK algorithm is $Q\left(\alpha^{*}\right)=11.3734$

So, we obtain a set of nonlinear equations including 15 parameters and 13 equations.

Moreover, by comparing the coefficients of $h^{5}$ in (4) and (14), we can get the local truncation error $E=h^{5}\left(\zeta_{1} D^{4} f+\zeta_{2} D f D^{2} f_{y}+\zeta_{3} D^{2} f D f_{y}+\zeta_{4} D^{2} f f_{y}^{2}+\zeta_{5} D f f_{y}^{3}+\zeta_{6}(D f)^{2} f_{y y}+\zeta_{7} D^{3} f f_{y}+\zeta_{8} f_{y} D f D f_{y}\right)+\cdots$

Among which the coefficients of local truncation error are

$$
\begin{aligned}
& \zeta_{1}=\frac{c_{2} a_{2}^{4}+c_{3} a_{3}^{4}+c_{4} a_{4}^{4}+c_{5} a_{5}^{4}-\frac{1}{24}}{120} \\
& \zeta_{2}=\frac{c_{3} b_{32} a_{2} a_{3}^{2}+c_{4}\left(b_{42} a_{2}+b_{43} a_{3}\right) a_{4}^{2}+c_{5}\left(b_{52} a_{2}+b_{53} a_{3}+b_{54} a_{4}\right) a_{5}^{2}}{2}-\frac{1}{20} \\
& \zeta_{3}=\frac{c_{3} b_{32} a_{2}^{2} a_{3}+c_{4}\left(b_{42} a_{2}^{2}+b_{43} a_{3}^{2}\right) a_{4}+c_{5}\left(b_{52} a_{2}^{2}+b_{53} a_{3}^{2}+b_{54} a_{4}^{2}\right) a_{5}}{2}-\frac{1}{30} \\
& \zeta_{4}=\frac{c_{4} a_{2}^{2} b_{32} b_{43}+c_{5}\left[a_{2}^{2} b_{32} b_{53}+\left(a_{2}^{2} b_{42}+a_{3}^{2} b_{43}\right) b_{54}\right]}{2}-\frac{1}{120} \\
& \zeta_{5}=c_{5} a_{2} b_{32} b_{43} b_{54}-1 / 120 \\
& \zeta_{6}=\frac{c_{3} a_{2}^{2} b_{32}^{2}+c_{4}\left(a_{2} b_{42}+a_{3} b_{43}\right)^{2}+c_{5}\left(a_{2} b_{52}+a_{3} b_{53}+a_{4} b_{54}\right)^{2}}{2}-\frac{1}{40} \\
& \zeta_{7}=\frac{c_{3} a_{2}^{2} b_{32}+c_{4}\left(a_{2}^{3} b_{42}+a_{3}^{3} b_{43}\right)+c_{5}\left(a_{2}^{3} b_{52}+a_{3}^{3} b_{53}+a_{4}^{3} b_{54}\right)^{2}}{2}-\frac{1}{120} \\
& \zeta_{8}=c_{4} a_{2}\left(a_{3}+a_{4}\right) b_{32} b_{43}+c_{5}\left[a_{2}\left(a_{3}+a_{5}\right) b_{32} b_{53}+\left(a_{4}+a_{5}\right)\left(a_{2} b_{42}+a_{3} b_{43}\right) b_{54}\right]-7 / 120
\end{aligned}
$$

Taking the local truncation error function as

$$
B=B(\zeta)=\sum_{i=1}^{8}\left|\zeta_{i}\right|
$$

The optimal parametric value and the related target function value can be obtained by simulated annealing algorithm

$$
c_{5}^{*}=0.5757, \quad b_{54}^{*}=0.2760, \quad B\left(c_{5}^{*}, b_{54}^{*}\right)=0.0072
$$

Finally, the new real-time RK4 algorithm formula is

$$
\left\{\begin{aligned}
y_{n+1} & =y_{n}+h\left(0.1174 k_{1}-0.0112 k_{2}+0.746 k_{3}-0.4279 k_{4}+0.5757 k_{5}\right) \\
k_{1} & =f\left[t_{n}, y_{n}\right] \\
k_{2} & =f\left[t_{n}+1 / 5 * h, y_{n}+1 / 5 h k_{1}\right] \\
k_{3} & =f\left[t_{n}+2 / 5 * h, y_{n}-0.00676 h k_{1}+0.40676 h k_{2}\right] \\
k_{4} & =f\left[t_{n}+3 / 5 * h, y_{n}+0.01628 h k_{1}+0.236 h k_{1}+0.34772 h k_{3}\right] \\
k_{5} & =f\left[t_{n}+4 / 5 * h, y_{n}+0.2554 h k_{1}-0.24749 h k_{2}+0.51613 h k_{1}+0.27597 h k_{4}\right]
\end{aligned}\right.
$$

\section{Example and conclusions}

Suppose an initial value problem

$$
\left\{\begin{array}{l}
\dot{y}=\frac{1}{2} x+\frac{1}{2} y, \quad x \in[0,0.6] \\
y(0)=1
\end{array}\right.
$$

Of which the precise solution is $y(x)=3 e^{0.5 x}-x-2$.

Here we take step $h=0.1$ and the result is listed in Table 1. Error $=y\left(t_{\mathrm{n}}\right)-y_{\mathrm{n}}$, double-precision is adopted. For comparison the result of classical RK4 formula is also listed.

It's clear from Table 1 that the result of our formula is superior to that of classical RK4 formula, has better accuracy and is suitable to real-time simulation. The example shows that the deduction of parametric equations and the result are correct and the calculation of the coefficients of local truncation errors is accessible, so the new real-time RK4 algorithm may have more extensive applications. 
Table $1 h=0.1$, simulation error of example 1

\begin{tabular}{lll}
\hline$t_{\mathrm{n}}$ & Error (RK4) & Error (this paper) \\
\hline 0.1 & $7.8780719725557 \mathrm{e}-009$ & $3.66458885459053 \mathrm{e}-009$ \\
0.2 & $1.6563978633144 \mathrm{e}-008$ & $7.70495245383529 \mathrm{e}-009$ \\
0.3 & $2.6119848950401 \mathrm{e}-008$ & $1.21499916794221 \mathrm{e}-008$ \\
0.4 & $3.6612055609452 \mathrm{e}-008$ & $1.70305793911751 \mathrm{e}-008$ \\
0.5 & $4.8111495010161 \mathrm{e}-008$ & $2.23796952081301 \mathrm{e}-008$ \\
0.6 & $6.0693868375594 \mathrm{e}-008$ & $2.82325516298698 \mathrm{e}-008$ \\
\hline
\end{tabular}

\section{Acknowledgement}

Guangxi Natural Science Foundation(No.2013GXNSFAA019021).

\section{References}

[1] Y M. Chen and C. X. Yu, "Optimal Algorithm of Strongly Stable Runge-Kutta in Second Order". Journal of Yanshan University, vol. 26, no. 1, pp. 17-19, 2002.

[2] Y. Wu and X. H. Wu, "Optimal Algorithm of Strongly Stable Runge-Kutta in Three-step Order". Mathematica Applicata, vol. 18, no. s1, pp. 57-61, 2005.

[3] M. X. He, "Analysis on a Class of New Algorithm of Fourth Order R-K Method". College Mathematics, vol. 20, no. 1, pp. 72-74, 2006.

[4] Q. Y. Li, N. C. Wang and D. Y. Yi, Contemporary Numerical Analysis. Beijing: Higher Education Press, 1995.

[5] Ralston A. A first course in numerical analysis. Mc GrawHall, New York, 1966.

[6] M. Jiang and Zhen Q. Huang. "Real-time RK4 Formula with Maximum Stability Region". Journal of System Simulation, vol. 18, no. 2, pp. 306-308, 2006.

[7] M. Tanaka. "Runge-Kutta formulas with the ability of error estimation". Rep. Stat. Appl. Res. JUSE, vol. 13, no. 3, pp. 42-61, 1966. 